\title{
The effects of tamoxifen and estradiol on myometrial differentiation and organization during early uterine development in the CD1 mouse
}

\author{
Mohamed K Mehasseb, S C Bell and M A Habiba \\ Reproductive Sciences Section, Department of Cancer Studies and Molecular Medicine, University of Leicester, \\ Robert Kilpatrick Clinical Sciences Building, Leicester LE2 7LX, UK \\ Correspondence should be addressed to M K Mehasseb; Email: mkm7@le.ac.uk
}

\begin{abstract}
We used a neonatal mouse model to examine the histogenesis of uterine adenomyosis, and to test whether adenomyosis is due to an abnormality in myometrial differentiation, or in extracellular matrix proteins expression. We also studied the effects of tamoxifen and estradiol on uterine development, myometrial differentiation, and organization. Female CD1 pups were treated with oral tamoxifen $(1 \mathrm{mg} / \mathrm{kg})(n=27)$ or estradiol $(0.1 \mathrm{mg} / \mathrm{kg})(n=24)$ from age 1 to 5 days. Uteri from control $(n=27)$ and treated mice were obtained on days 2, 5, 10, 15, and 42 of age. We examined the sections histologically, using image analysis and immunohistochemistry for $\alpha$-smooth muscle actin ( $\alpha$-SMA), desmin, vimentin, laminin, fibronectin, and estrogen receptor- $\alpha$. Following tamoxifen exposure, all uteri showed adenomyosis by 6 weeks of age (seen as early as day 10). The inner myometrium showed thinning, lack of continuity, disorganization, and bundling. $\alpha$-SMA expression was normal. Desmin expression normally showed a wave of maturation that was absent in tamoxifen-treated mice. In the estradiol group, adenomyosis was not observed. All uterine layers were normally developed, but hypertrophied. The inner myometrium retained its circular arrangement. There was no difference in the localization of laminin or fibronectin between groups (laminin expression was reduced in the tamoxifen treated uteri). Vimentin could not be detected in all groups. Our results suggest that the development of the inner myometrium is particularly sensitive to estrogen antagonism, and can be affected by steroid receptors modulation. Disruption of the inner myometrium may play a role in the development of uterine adenomyosis.

Reproduction (2009) 138 341-350
\end{abstract}

\section{Introduction}

Uterine adenomyosis is characterized by the presence of endometrium within the myometrium. Microscopically, ectopic, non-neoplastic, endometrial glands, and stroma appear surrounded by hypertrophic and hyperplastic myometrium (Bird et al. 1972). Adenomyosis has been associated with abnormal uterine bleeding, dysmenorrhoea, and chronic pelvic pain. The percentage of hysterectomy specimens containing adenomyosis varies from 5 to $70 \%$ (Azziz 1989). Risk factors for adenomyosis in women include age, parity, and tamoxifen use. Sharp curettage during early pregnancy has also been linked to the development of adenomyosis, which suggests that the disruption of the endometrial-myometrial interface may be important in the pathogenesis.

Spontaneously occurring adenomyosis has been reported in several animal species including nonhuman primates, dogs, cats, and laboratory rodents and rabbits (McCann \& Myers 1970, Suire et al. 1978).
In CD1 mice, spontaneous adenomyosis starts to appear from about 6 months of age onwards, and over $80 \%$ are affected by 12 months of age, disease severity increases thereafter (Greaves \& White 2006). Various forms of hormonal manipulation can enhance the development of adenomyosis in the mouse. These include hyperprolactinemia (Huseby \& Thurlow 1982, Mori \& Nagasawa 1983), prolonged estrogen (Guttner 1980), or progesterone administration (Ostrander et al. 1985).

One experimental model involved transplant of the anterior pituitary in the lumen of adult SHN and SLN mouse strains (Mori \& Nagasawa 1983, Mori et al. 1991). Almost all animals developed adenomyosis in both uterine horns. Adenomyosis was also reported following neonatal administration of prolactin or dopamine antagonists (Mori et al. 1981). Prolonged exposure of BALB/C mice to progesterone, norethisterone, or norethynodrel increased the incidence of adenomyosis (Lipschutz et al. 1967). Prolonged progesterone treatment following diethylstilboestrol also induced adenomyosis in ovariectomized mice, 
suggesting a link between progesterone and the development of adenomyosis (Ostrander et al. 1985). On the other hand, estrogen unopposed by progestogen administered to ovariectomized rhesus monkeys induced adenomyosis (Baskin et al. 2002).

More recently, adenomyosis has been demonstrated in CD1 mice following neonatal administration of tamoxifen (Parrott et al. 2001, Green et al. 2005). Oral administration of tamoxifen $(200 \mu \mathrm{g} / \mathrm{ml}$, at a dose $5 \mu \mathrm{l} / \mathrm{g}$ body weight) to female pups between days 1 and 5 of age induced adenomyosis in all uteri examined at 6 weeks postnatal. This was also associated with marked disorganization of the myometrium and the mesenchyme. The ovaries of the adult mice were noted to be normal and to contain corpora lutea, and as early uterine development was not assessed, the possibility remains that ovarian steroids may have a role in the pathogenesis of adenomyosis. It is also interesting to note that adenomyosis was not induced following the administration of an equivalent uterotropic dose of estradiol, or following the administration of raloxifene that has no uterotropic effect.

The mesenchyme surrounding the developing Mullerian duct gives rise to $90-95 \%$ of the uterine mass and forms both the myometrium and the endometrial stroma (Martin et al. 1973), and has been shown to express estrogen receptor (ER). In contrast to the epithelium that expresses ER at postnatal days 6-7, Mullerian duct mesenchyme in the CD1 mouse expresses ER from day 13 of gestation (Eide 1975, Stumpf et al. 1980, Holderegger \& Keefer 1986).

Esr1 mRNA was found in CD1 mouse uterus as early as fetal day 14; by contrast, low-level expression of Esr2 mRNA was only detected on the first postnatal day. However, while ESR1 was detected using immunohistochemistry in stromal cells during late gestation and the postnatal period (Jefferson et al. 2000, Kurita et al. 2001), ESR2 was not detected before postnatal day 6 (Jefferson et al. 2000, Kurita et al. 2001, Parrott et al. 2001).

In this study, we used this model to examine the histogenesis of uterine adenomyosis. We compared normal uterine development and morphogenesis with that following tamoxifen and estradiol administration. We then examined the effect of tamoxifen and estradiol on the developing uterus, as manifested by changes in the patterns of expression of cytoskeletal proteins (actin, desmin, and vimentin), extracellular matrix proteins (laminin and fibronectin), and ESR1. We examined the hypothesis that adenomyosis and the associated muscle changes are due to an abnormality in myometrial differentiation, or in the extracellular matrix proteins expression. We also examined the possible involvement of ER and its regulation by tamoxifen and estradiol, in relation to the development of adenomyosis.

\section{Results}

In the following subsections, we first present the normal uterine development in the CD1 mice. Then, we describe the morphological changes observed in the tamoxifen- and estradiol-treated groups. We finally present our data on immunohistochemical markers. The data are visually presented and summarized in Figs $1-4$ and Tables 1 and 2.

\section{Morphology and uterine development}

Normal uterine development of CD1 mice

Day 2. The uterine cavity consisted of an oval-shaped lumen, elongated in the mesometrial-antimesometrial axis. The luminal epithelium consisted of a monolayer of low columnar cells, with large round nuclei and prominent nucleoli. The uterine wall thickness was formed of mesenchymal cells with large pale cytoplasm, large round nuclei, and less prominent nucleoli. These cells were not differentiated into distinct layers or orientation. No discernible blood vessels could be seen. The perimetrium was composed of a single layer of cuboidal to low columnar epithelium.

Day 5. The monolayer luminal epithelium consisted of tall columnar cells with elongated nuclei arranged in palisade with some multilayered areas. There was no evidence of gland formation. In the uterine wall, the mesenchymal cells started to segregate into three layers: endometrial stroma, inner circular, and prospective outer longitudinal muscle layers. The endometrial stroma occupied the inner half of the uterine wall thickness. The stromal cells retained their undifferentiated shape and loose arrangement with no particular spatial orientation. The prospective inner circular muscle layer was the most defined and densely staining layer, five to six cells thick. The cells were bundled, circularly orientated, and tightly packed with elongated nuclei and minimal intercellular space. The prospective outer myometrium was formed of one to two layers of cells retaining their undifferentiated appearance. Vascular spaces started to appear, especially in the layer between the inner and outer myometrium.

Day 10. Uterine sections showed a more complex luminal epithelium invaginating the stroma to form simple tubular uterine glands. All layers of the uterine wall were more distinct. The stroma appeared more tightly packed and surrounded the endometrial glands. The inner circular muscle layer was thicker compared with the outer myometrium. The cells were organized into bundles with no surrounding connective-tissue sheath. Longitudinally cut sections demonstrated elongated nuclei in this outer layer, confirming its longitudinal arrangement. Blood vessels were larger and observed in all layers of the uterine wall. 


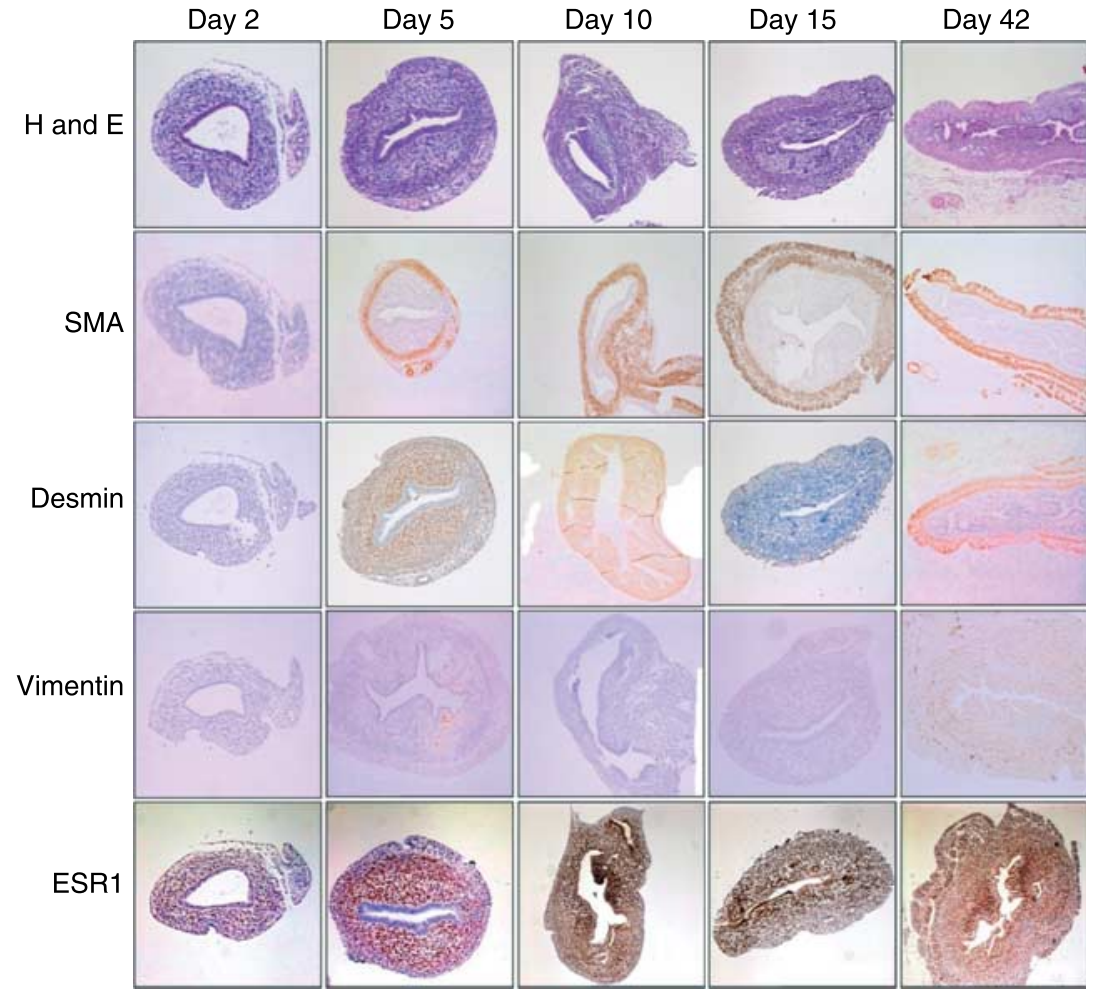

Figure 1 Control CD1 mice: uterine development and immunohistochemical staining pattern for $\alpha$-smooth muscle actin, desmin, vimentin, and ESR1. Magnification: days 2, 5, 10, and 15 micrographs $(\times 20)$, day $42(\times 10)(H$ and $E$, hematoxylin and eosin; SMA, $\alpha$-smooth muscle actin).
Day 15. The adult configuration of the uterus was apparent. The endometrium showed numerous simple tubular glands and a thick endometrial stroma. Stromal cells were randomly orientated except around the individual glands where they appeared more circular. A distinct loose vascular layer separated the inner and outer myometrial layers. The outer myometrial cells became grouped in bundles connected by loose connective tissue sheaths, and separated from the inner myometrium by a distinct loose vascular layer.

Day 42. The adult uterus was larger and more complex, but had the same configuration as the uterus on day 15 . The endometrial glands were more complex and branching, but were confined to the endometrial layer. The inner myometrium was quite distinct. The intervening vascular layer harbored most of the large blood vessels. The outer longitudinal muscle layer was formed of prominent bundles with well-formed connective tissue sheaths.

\section{Uterine development following tamoxifen administration}

Day 2. The uterine epithelium was hypertrophied, with large nuclei and prominent nucleoli. The mesenchymal cells in the uterine wall were uniform in shape and size, and did not show any specific spatial arrangement.

Day 5. The uterine lumen was markedly dilated with the prominent secretions, and the uteri were significantly heavier than controls. The hypertrophied epithelium was formed of tall columnar cells with pale elongated nuclei. Blood-filled spaces were seen throughout the uterine wall, which was thinner compared with controls. Some cells in the middle of the uterine wall appeared to adopt circular orientation, but these were loosely bundled and only formed a thin patchy ring encircling about $25-40 \%$ of the uterus. One section examined revealed deep glands involving $>30 \%$ of the uterine thickness, representing early evidence of adenomyosis.

Day 10. The uterus was dilated and adenomyosis was observed in three out of five specimens. There was marked disorganization of uterine development, with glands dispersed all through the uterine wall, but not reaching the serosa. The original lumen was sometimes hard to identify. The stroma surrounding the glands was highly cellular with ovoid nuclei. The glandular epithelium was less hypertrophied. Otherwise, the myometrial layers were not distinct. Some cells had circular orientation, but with no observable continuity and with some glands seen deeper to the circular layer. The outer muscle layer was less obvious, with some bundles observed on the periphery.

Day 15. Adenomyosis was observed in $80 \%$ of specimens. The same earlier disorganized development pattern was observed. The circular muscle layer disruption was more evident as cells acquired darker staining cytoplasm and more obvious circular orientation, and some glands extending deeper to the 
circular muscle layer. The outer myometrium showed signs of differentiation into bundles surrounded by thin loose connective tissue. The intervening vascular layer was not obvious, although some prominent blood vessels were seen scattered in the uterine wall.

Day 42. Adenomyosis was observed in all the specimens to various degrees. The uteri were smaller than controls. Some glands reached the serosa forming subserosal cysts. The endometrial stroma was highly cellular. The circular myometrium showed marked loosening and increased intercellular space. The outer myometrium was well developed with the obvious bundling. Overall, the muscle integrity was more preserved on the antimesometrial border of the uterus.

\section{Uterine development following estradiol administration}

In general, the uterine development in estradiol-treated CD1 mice was similar to the control mice, but development and appearance of the different layers were observed at earlier chronological stages. By day 2, the cells in the middle third of the uterine wall have already demonstrated a clear circular arrangement with the dark cytoplasm staining. By day 5, the circular muscle layer was well developed, with tightly packed bundles of dark cells, and with minimal intercellular space. The uterine wall contained many blood-filled spaces. Later on, the uterine layers were well developed and easily identified. The uterine size was generally larger compared with the control counterpart, but the different layers retained their proportions and relative sizes. Endometrial glands were abundant. There was no evidence of adenomyosis or myometrial disruption.

\section{Immunohistochemical markers}

\section{$\alpha$-Smooth muscle actin expression}

On day 2 , control uteri were negative for $\alpha$-smooth muscle actin $(\alpha-S M A)$. A narrow but distinct zone of variable intensity staining corresponding to the developing inner circular myometrium was seen in the middle of the uterine wall by day 5 . On day $10, \alpha$-SMA expression was strong and well defined in the inner circular 'muscle' layer; less intense expression was seen in the outer longitudinal muscle layer. Uteri from day 15 onwards exhibited well-defined, intense staining of both the inner and outer muscle layers.

In the tamoxifen group, $\alpha$-SMA expression was weaker, with fewer positive cells/area, reflecting disruption and loosening of the circular myometrium. Staining intensity was generally weaker in the inner circular compared with the outer longitudinal muscle layers.

In estradiol-treated mice, positive immunostaining for $\alpha$-SMA was observed as early as day 2 in the middle section of the uterine wall. The staining was generally intense particularly in the compact thickened circular myometrium.

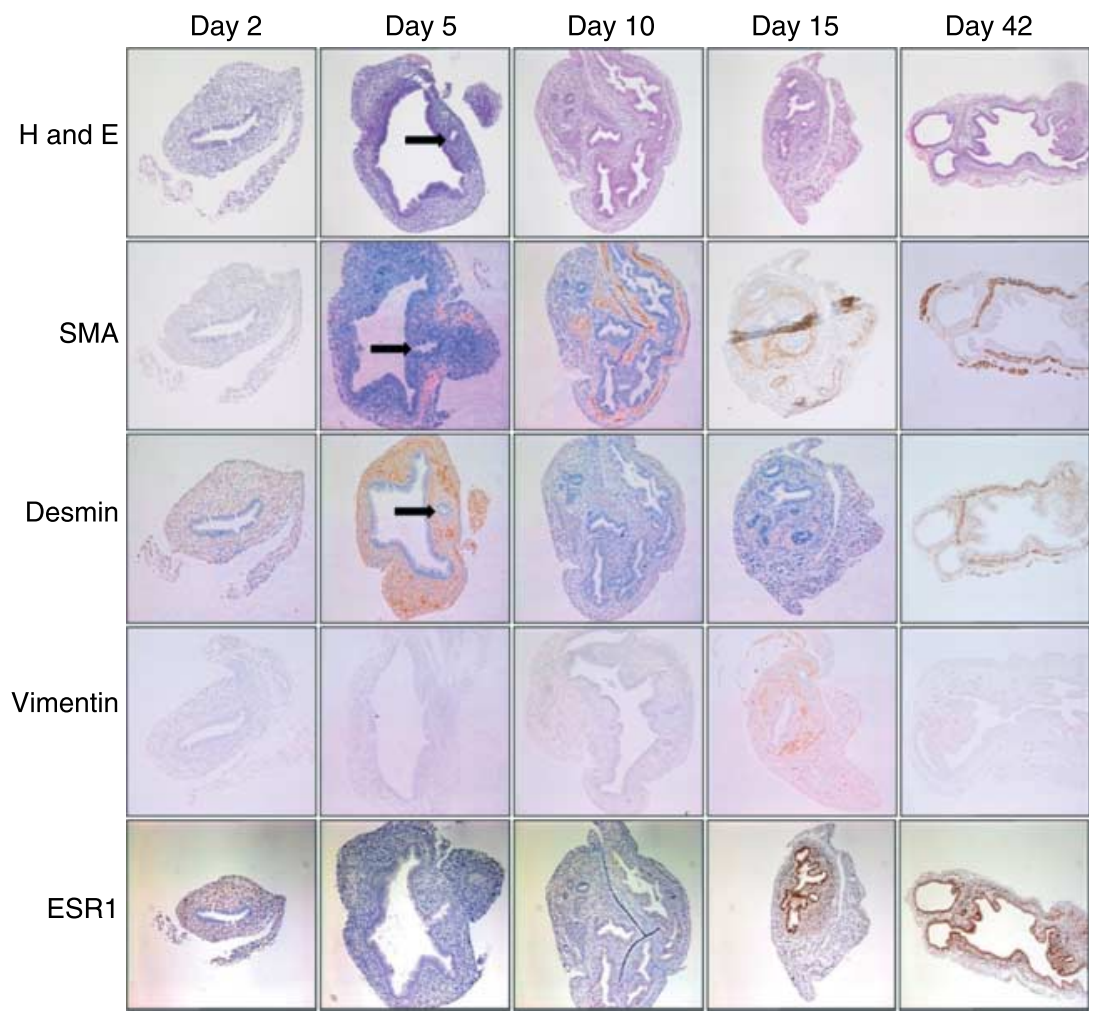

Figure 2 Tamoxifen-treated CD1 mice: uterine development and immunohistochemical staining pattern for $\alpha$-smooth muscle actin, desmin, vimentin, and ESR1. Magnification: days 2, 5, 10, and 15 micrographs $(\times 20)$, day $42(\times 10)$. Black arrow points to early invading glands (day 5 ), leading to adenomyosis (day 10). Note the marked disorganization and disruption of the inner myometrium around the adenomyosis foci. Subserosal adenomyotic cysts are evident on day 42. Also note the reduced desmin expression in the myometrial layers ( $\mathrm{H}$ and $\mathrm{E}$, hematoxylin and eosin; SMA, $\alpha$-smooth muscle actin). 


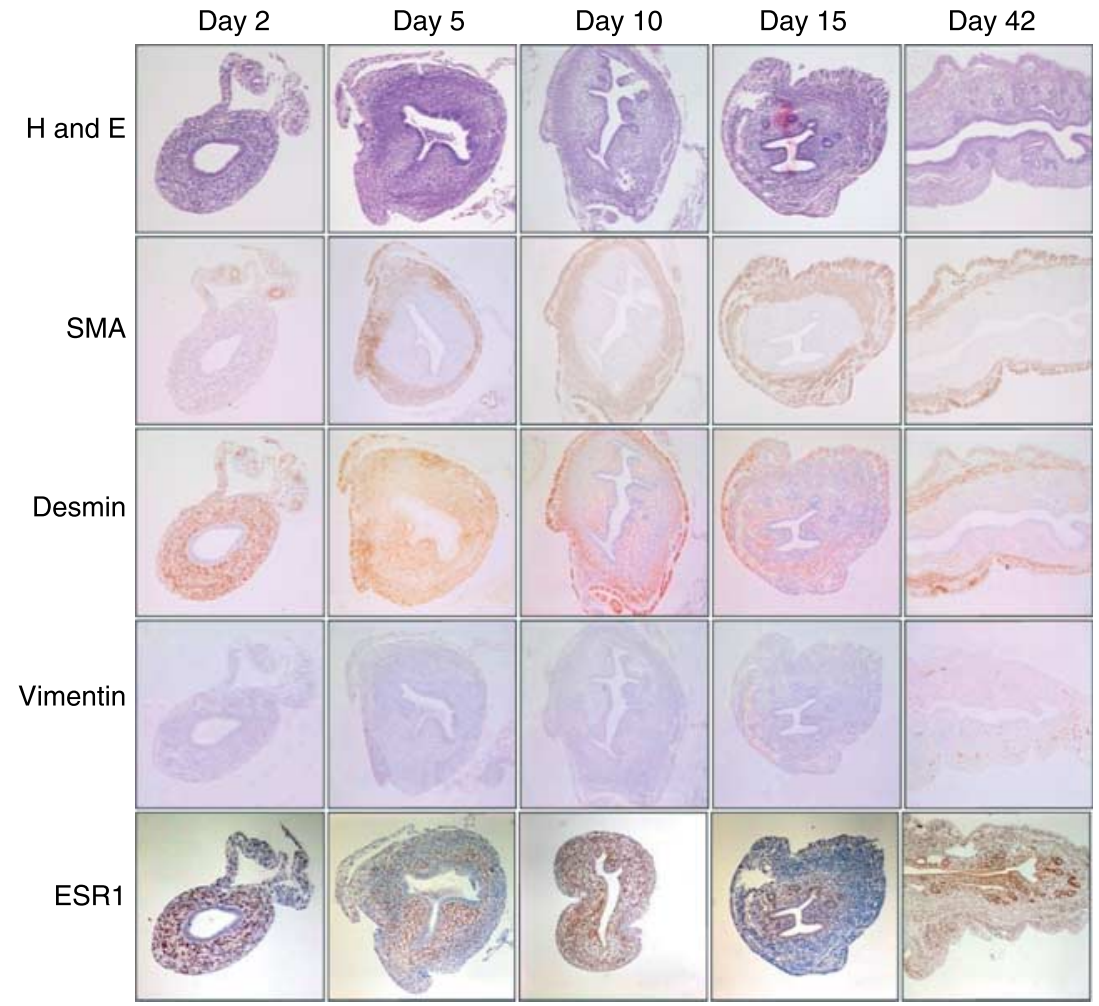

Figure 3 Estradiol-treated CD1 mice: uterine development and immunohistochemical staining pattern for $\alpha$-smooth muscle actin, desmin, vimentin, and ESR1. Magnification: days 2, 5, 10 , and 15 micrographs $(\times 20)$, day $42(\times 10)$ ( $\mathrm{H}$ and $\mathrm{E}$, hematoxylin and eosin; SMA, $\alpha$-smooth muscle actin).

\section{Desmin expression}

In control CD1 mice, desmin expression followed a 'wave' of maturation from inside outwards. Firstly, on day 2 , the whole uterine thickness was weakly immunopositive to desmin. The developing endometrial stroma then strongly expressed desmin, while the outer and middle parts of the uterine wall remained less intense. As the outer and longitudinal myometria developed, they acquired and expressed stronger immunostaining to desmin compared with the endometrial stroma that reverted to be weakly positive. The blood-vessels walls stained strongly positive, while endometrial epithelium was negative.

In tamoxifen-treated mice, this wave of maturation was absent. The intensity of staining remained uniformly weak all through the developmental phases of the uterine wall structures. Generally, the intensity of staining was weaker than in controls. However, in the adult uterus (day 42), the myometrial layers showed relatively stronger staining compared with the endometrial stroma. The endometrial epithelium remained negative.

Estradiol-treated mice demonstrated an earlier (day 2) stronger desmin immunostaining. The undifferentiated uterine wall strongly expressed desmin and the early developing myometrial layers were more intense than the endometrial stroma. The endometrial epithelium stained negatively for desmin. All blood-vessels' walls stained strongly positive and were considered internal quality control for the immunostaining.

\section{Vimentin expression}

Vimentin immunohistochemical expression was observed in the endometrial stroma and blood-vessel wall starting on day 15 and day 42 (in all groups). No staining was seen in the myometrium. In some uteri, occasional staining was observed in the inner part of the

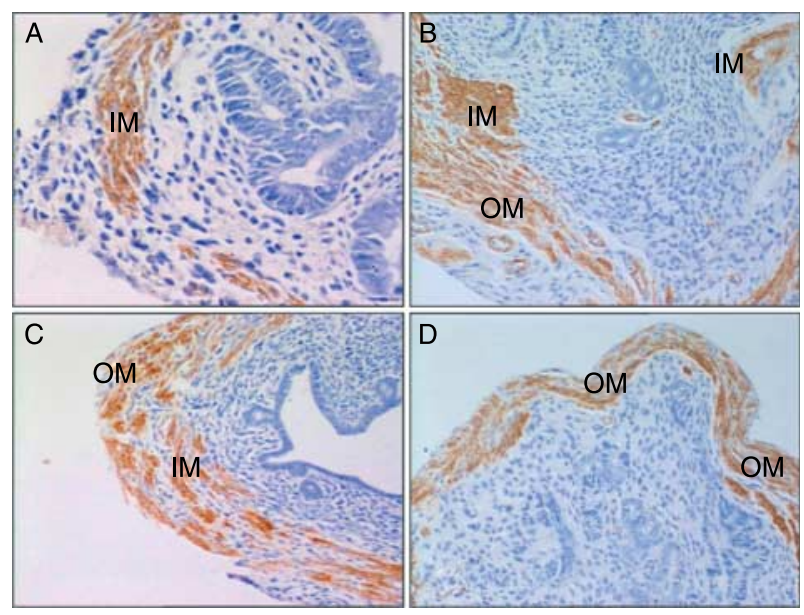

Figure $4 \alpha$-Smooth muscle actin immunostaining, demonstrating the myometrial abnormalities associated with the presence of uterine adenomyosis. (A) Bundling and loosening of the inner myometrium. (B) Disruption of the inner myometrium. (C) Increased extracellular space. (D) Normal outer myometrium immunostaining (IM, inner myometrium; OM, outer myometrium) $(\times 40)$. 
Table 1 Histological features measured at different stage of development of neonatal CD1 mice after administration of tamoxifen, estradiol, or vehicle only. Measurements are in $\mu \mathrm{m}$ : median (range).

\begin{tabular}{|c|c|c|c|}
\hline & Control & Tamoxifen $^{\mathrm{a}}$ & Estradiol $^{b}$ \\
\hline \multicolumn{4}{|l|}{ Day $2(n=5)$} \\
\hline Epithelial height & $14(10-16)$ & $14(13-15)$ & $12(10-15)$ \\
\hline Uterine-wall thickness & $60(40-75)$ & $70(60-75)$ & $70(60-75)$ \\
\hline \multicolumn{4}{|l|}{ Day $5(n=5)$} \\
\hline Epithelial height & $18(16-20)$ & $25(25-35)$ & $20(18-25)$ \\
\hline Uterine-wall thickness & $110(100-110)$ & $70(60-80)$ & $90(85-110)$ \\
\hline Inner cellular layer (future stroma) & $70(60-80)$ & $35(35-40)$ & $50(45-60)$ \\
\hline Inner circular muscle layer & $30(25-35)$ & $20(20-25)$ & $30(25-35)$ \\
\hline Outer cellular layer (future outer myometrium) & $12(10-15)$ & $12(10-15)$ & $20(16-22)$ \\
\hline \multicolumn{4}{|l|}{ Day $10(n=5)$} \\
\hline Epithelial height & $18(16-20)$ & $15(12-20)$ & $16(15-18)$ \\
\hline Uterine-wall thickness & $120(110-130)$ & $300(270-310)^{\mathrm{c}}$ & $120(110-130)$ \\
\hline Inner cellular layer (future stroma) & $90(80-100)$ & $-^{d}$ & $90(80-100)$ \\
\hline Inner circular muscle layer & $30(25-35)$ & $-^{d}$ & $30(25-35)$ \\
\hline Outer cellular layer (future outer myometrium) & $20(15-25)$ & $-{ }^{d}$ & $20(15-25)$ \\
\hline \multicolumn{4}{|l|}{ Day $15(n=5)$} \\
\hline Epithelial height & $18(15-20)$ & $18(15-20)$ & $18(16-20)$ \\
\hline Uterine-wall thickness & $170(160-180)$ & $330(300-340)^{c}$ & $150(140-165)$ \\
\hline Endometrial stroma & $100(90-110)$ & $-{ }^{d}$ & $80(75-90)$ \\
\hline Inner circular myometrium & $40(35-40)$ & $-{ }^{d}$ & $35(30-40)$ \\
\hline Intermediate vascular layer & $10(10-15)$ & $-^{\mathrm{d}}$ & $10(10-15)$ \\
\hline Outer myometrium & $25(20-30)$ & $25(20-30)$ & $30(20-35)$ \\
\hline \multicolumn{4}{|l|}{ Day $42(n=5)$} \\
\hline Epithelial height & $18(15-20)$ & $20(15-20)$ & $18(16-20)$ \\
\hline Uterine-wall thickness & $340(320-360)$ & $300(280-310)^{\mathrm{C}}$ & $550(450-600)$ \\
\hline Endometrial stroma & $120(110-130)$ & ${ }_{-}^{\mathrm{d}}$ & $160(140-300)$ \\
\hline Inner circular muscle layer & $80(75-90)$ & $-^{\mathrm{d}}$ & $100(80-120)$ \\
\hline Intermediate vascular layer & $50(50-55)$ & $-^{\mathrm{d}}$ & $50(45-60)$ \\
\hline Outer myometrium & $90(85-100)$ & $50(40-60)$ & $110(100-120)$ \\
\hline
\end{tabular}

${ }^{a}$ The uteri in the tamoxifen group had wide irregular lumens and poorly defined layers characterized by penetrating stroma, markedly disrupted circular layer, and no obvious vascular layer. ${ }^{\mathrm{b}}$ Estradiol treated uteri had distinct well-developed layers starting from day 2 . $^{\mathrm{C}}$ Uterine lumen was not recognizable from adjacent adenomyotic glands in the tamoxifen group. Uterine wall thickness was measured as half the diameter of the uterus. ${ }^{\mathrm{d}}$ Glands and surrounding stroma were interspersed with disrupted muscle bundles and blood vessels. The stromal layer, inner circular and intermediate vascular layers were not distinctly identifiable and could not be measured separately.

uterine wall (developing stroma) by day 5 , but this was not consistent. All blood-vessels' walls stained positive and were considered intrinsic quality control.

\section{Laminin and fibronectin expressions}

The undifferentiated mesenchyme showed light diffuse expression for laminin and fibronectin on day 2. By day 5, laminin expression was more localized to the middle third of the developing uterus, i.e. the prospective inner circular muscle layer, while fibronectin was differentially expressed in the prospective endometrial stroma (inner third of the developing uterus). The same expression pattern was observed on day 10, and subsequently, as laminin was exclusively expressed in the extracellular matrix surrounding the smooth muscle layers (inner circular and outer longitudinal). Fibronectin was exclusively expressed around the stromal cells and in the connective tissue sheaths separating the outer longitudinal muscle and the intervening vascular layer.

There was no difference in the localization of laminin or fibronectin between the controls and treatment groups. However, laminin expression was reduced in the tamoxifen-treated uteri, secondary to the disruption of the inner myometrium, and on day 5 possibly secondary to tissue oedema.

\section{ESR1 expression}

In the control CD1 mice, the whole thickness of the uterine wall expressed ESR1 from day 2, while the epithelial lining remained negative till day 10, when both luminal and glandular epithelia exhibited strong positive staining. As the various layers developed in the mesenchyme, the ESR1 expression was more intense in the stroma compared with the muscle layers. By day 42, there was more variability in staining intensity between the specimens, due to different phases of the estrous cycle.

In tamoxifen-treated mice, there was marked downregulation of the ESR1, with no or very weak staining in all the layers in days 5 and 10 . However, by day 15 , the ESR1 staining has recovered in the endometrial layers (epithelium and stroma), but was less obvious in the myometrium. On day 42, the glands and stroma were strongly positive (including in adenomyotic foci), but the muscle layers (inner and outer) remained less 
Table 2 Immunohistochemical expression of desmin and vimentin in the uterus CD1 mice. The control group received vehicle only, study group received tamoxifen or estradiol from neonatal days 1 to 5 . Uteri were examined on day 2, 5, 10, 15, and 42 of age.

\begin{tabular}{|c|c|c|c|c|c|c|}
\hline & \multicolumn{2}{|c|}{ Control } & \multicolumn{2}{|c|}{ Tamoxifen } & \multicolumn{2}{|c|}{ Estradiol } \\
\hline & Desmin & Vimentin & Desmin & Vimentin & Desmin & Vimentin \\
\hline \multicolumn{7}{|l|}{ Day $2(n=5)$} \\
\hline Uterine wall & + & - & + & - & +++ & - \\
\hline \multicolumn{7}{|l|}{ Day $5(n=5)$} \\
\hline Inner layer & +++ & \pm & + & - & ++ & - \\
\hline Middle layer & + & - & + & - & \pm & - \\
\hline Outer layer & + & - & + & - & $\overline{+}$ & - \\
\hline \multicolumn{7}{|l|}{ Day $10(n=5)$} \\
\hline Stroma & + & - & \pm & - & + & - \\
\hline Inner myometrium & ++ & - & \pm & - & ++ & - \\
\hline Outer myometrium & + & - & \pm & - & ++ & - \\
\hline \multicolumn{7}{|l|}{ Day $15(n=5)$} \\
\hline Stroma & \pm & + & + & + & + & + \\
\hline Inner myometrium & ++ & - & + & - & ++ & - \\
\hline Outer myometrium & ++ & - & + & - & ++ & - \\
\hline \multicolumn{7}{|l|}{ Day $42(n=7)$} \\
\hline Stroma & \pm & + & + & + & + & + \\
\hline Inner myometrium & $+\overline{+}+$ & - & ++ & - & +++ & - \\
\hline Outer myometrium & +++ & - & ++ & - & +++ & - \\
\hline
\end{tabular}

immunopositive. Overall, ESR1 expression was attenuated compared with control uteri. In estradiol-administered mice, ESR1 expression was comparable to control mice, although on day 5 and 10 ESR1 was transiently over expressed in all the layers.

\section{Discussion}

At birth, the uterus of CD1 mice was made of simple low columnar epithelium supported by undifferentiated mesenchyme, and lacked endometrial glands. Uterine development involved the differentiation and development of the endometrial glands from the luminal epithelium and the inner circular and outer longitudinal layers of muscle from the uterine mesenchyme. The described developmental stages in our study are in agreement with the previous reports using CD1 strain (Cunha 1976, Brody \& Cunha 1989, Cunha et al. 1992).

Perinatal uterine development represents a critical phase for uterine morphogenesis and differentiation (Iguchi \& Sato 2000). This model demonstrated the early stages of development of uterine adenomyosis, in the absence of progestogenic effect. Tamoxifen, but not estradiol neonatal exposure, resulted in the development of adenomyosis in CD1 mice as early as day 10, with the disruption of the inner myometrial layer and ingrowth of endometrial glands and stroma. The doses of tamoxifen and estradiol used in our work were derived from the dose-response studies (Green et al. 2001), and have been previously described and utilized in the similar experiments (Parrott et al. 2001, Green et al. 2003).

The exact genetic and environmental factors regulating cell line differentiation in the uterus are not fully known. The present study clearly shows that even in 5-days-old CD1 mice, tamoxifen has a uterotropic effect. Interaction of estrogen agonists with ESR1 plays a key role in the initial uterotrophic effect since immature ESR1 knockout mice, when given tamoxifen, show no significant increase in uterine weight (Korach 1994). The observed immunohistochemical changes are due to a direct action on the uterus, as the hypothalamic/ pituitary axis is inactive in this early stage. Gene expression arrays demonstrated similar changes in 14-days-old female mice uteri following estradiol, genistein, and diethylstilbestrol administration, which suggest that the actions of estrogens on the uterus are intrinsically similar (Moggs et al. 2004). The same conclusion cannot be drawn in relation to tamoxifen.

Epithelial-mesenchymal interactions are recognized to play an important role in the postnatal development and the spatial organization of the uterus (Cunha 1976, Cunha et al. 1989, 1992). $\alpha$-SMA and the intermediate filaments (desmin and vimentin) were used as differential markers for myocytes, myofibroblasts, and fibroblasts cellular differentiation, as they are tissue specific and their pattern of expression is developmentally regulated. Recent work has suggested that ESR2 could be involved in the differentiation process of stromal cells and fibroblasts into myofibroblasts in various breast tumors (Sapino et al. 2006). It is possible that tamoxifen could alter the paracrine signaling in the uterus (via a receptor-mediated mechanism), affecting the differentiation of uterine myocytes in the mesenchyme and, over a period of time, allowing invasion of the endometrium into the myometrium. However, our observations suggest that the developing myometrium expressed an immunohistochemical profile suggestive of myogenic differentiation (absence of vimentin and expression of $\alpha$-SMA). The histodifferentiation of smooth muscle cells was clearly demonstrated 
by $\alpha$-SMA staining (although delayed) in both the intact and disrupted inner muscle layers, and in the outer myometrium. This argues against the abnormal differentiation into various fibroblasts/myofibroblasts phenotypes.

The absence of vimentin immunostaining in the differentiating myometrial layers makes fibroblastic differentiation less likely. By contrast, those cells that were destined to form the endometrial stroma expressed vimentin. The absent myometrial expression of vimentin was not altered between control-, tamoxifen-, and estradiol-administered mice. The distribution and function of vimentin in the developing mouse myometrium are not well described in the literature. This suggests that vimentin may not be involved in the differentiation and development of myometrial cells in the early stages of mouse development. Similarly, vimentin reactivity could not be detected inside the myofibers of mice embryos or neonates (Li et al. 1997).

We observed reduced desmin expression in tamoxifen-administered mice. Desmin is expressed in all muscle tissue. Its role in both cardiac and skeletal muscle has been widely described (Lazarides 1980, Capetanaki et al. 1984, Li et al. 1997). However, little is known about its role and distribution in the myometrium. The fact that desmin is expressed very early in the muscle development suggests that it may play a modulating role in the myogenic differentiation (Lin et al. 1994, Li et al. 1997). Mice lacking desmin show severe disruption of the smooth muscle architecture, including loss of lateral alignment of myofibrils, perturbations of myofibrillar anchorage to the sarcolemma, abnormal mitochondrial number and organization, and loss of nuclear shape and positioning (Milner et al. 1996).

Our results are in agreement with the primary presence of desmin as an intermediate filament in the myogenic cells. A reduced desmin in tamoxifenadministered mice might point to a perturbation or dysfunction of the myofibers, leading to the weakness of its tensile strength and disruption with the resultant abnormal architecture. In skeletal muscles, desmin is essential for the tensile strength and integrity of myofibrils but not for myogenic commitment, differentiation, and fusion (Milner et al. 1996, Park et al. 2000, Shah et al. 2004).

In the uterus, the junction between the endometrium and the myometrium lacks a distinct basement membrane. Invasion of the endometrium into the underlying musculature may be due to endometrial or myometrial factors. In prolactin-induced adenomyosis, the myometrium becomes loose with increased intercellular space, and muscle cells become small and irregular. Disintegration of the muscle layer, with reduced cell organelles, and pyknosis of the myocytes were frequently seen in adenomyotic areas (Mori et al. 1984, Ohta et al. 1985). It is possible that the disruption of the muscle layer allows invasion by the overlying endometrium.
Adenomyosis development following tamoxifen treatment in CD1 mice could also be due to an alteration in the composition of the extracellular matrices, facilitating the 'invasion' of the endometrial stroma and glands into the myometrium. The invasive activity of the endometrial stromal cells into various kinds of extracellular matrix components was examined in experimentally induced adenomyosis in SHN mice, and it was suggested that the increased matrix metalloproteinase-14 may play an important role in the invasion of endometrial tissues into the myometrium (Matsuda et al. 2001). Laminins and fibronectins are important glycoprotein components of the extracellular matrix and basement membranes, and are critically involved in cell differentiation in early development and in tissue formation and maintenance of mature tissues (Romberger 1997, Sasaki et al. 2004). Our findings in control mice are in agreement with the previous reports (Brody \& Cunha 1989). The normal localization of laminin and fibronectin observed following tamoxifen treatment suggests that these molecules are unlikely to be the primary target for tamoxifen effect.

Our description of ESR1 expression in control uteri is in agreement with the previous studies (Kurita et al. 2001). ERs have been identified in the epithelium, stroma, and myometrium of the developing uterus. In CD1 mice, ER immunoreactivity was observed in stromal cells on day 1 , and in epithelial cells by day 5 (Yamashita et al. 1989, Sato et al. 1992). The mode of action of tamoxifen is complex, but our results demonstrated that tamoxifen significantly downregulates ESR1. Tamoxifen is able to antagonize the action of calmodulin, protein kinase $\mathrm{C}$, and compete for membrane-binding sites for neurotransmitter substances (Lam 1984, Kroeger \& Brandes 1985, Horgan et al. 1986, Batra 1990). Myometrial strips from non-pregnant human myometrium showed a marked inhibition of spontaneous and vasopressin-induced contractions when tamoxifen was added to the bathing solution (Kostrzewska et al. 1997). This spasmolytic activity on smooth muscle was also demonstrated in the rat myometrium, where tamoxifen antagonized the contractions induced by exogenous calcium (Lipton \& Morris 1986). It is thus plausible that the myometrial abnormalities observed following tamoxifen treatment could be related to an underlying dysfunction in the active processes involved in the contractile machinery and the regulation of calcium metabolism.

In conclusion, our results suggest that the development of the inner myometrium is particularly sensitive to estrogen antagonism, and can be affected by steroid receptors modulation. Tamoxifen - but not estradiol induces adenomyosis in neonatally administered CD1 mice by 6 weeks of age. Adenomyotic changes could be seen as early as day 10 , associated with the marked thinning, lack of continuity and orientation, disorganization and bundling of the inner myometrium. Desmin 
expression was markedly reduced. The endometrium and outer myometrium were not morphologically different. Thus, disruption of the inner myometrium may play a role in the development of uterine adenomyosis.

\section{Materials and Methods \\ Samples and materials}

The study was conducted under the authority of the United Kingdom Home Office, Animals (Scientific Procedures) Act 1986. Pregnant time-mated CD1 mice were obtained (Charles River Ltd, Margate, Kent, UK), and their female pups were divided into three groups. The first group $(n=27)$ was orally dosed on days 1-5 after birth (day of birth being day 0 ) with $1 \mathrm{mg} / \mathrm{kg}$ tamoxifen - suspended in peanut oil/lecithin/condensed milk mixture at a concentration of $200 \mu \mathrm{g} / \mathrm{ml}$, at a dose $5 \mu \mathrm{l} / \mathrm{g}$ body weight. The second group $(n=24)$ received $0.1 \mathrm{mg} / \mathrm{kg}$ estradiol benzoate - suspended in peanut oil/ lecithin/condensed milk mixture at a concentration of $20 \mu \mathrm{g} / \mathrm{ml}$, at a dose of $5 \mu \mathrm{l} / \mathrm{g}$ body weight. The control group $(n=27)$ received vehicle only. Five mice from each group were culled on days 2 (before the second dose), 5, 10, 15, and the remainder on day 42 . Uteri were collected, weighed, and fixed in $4 \%$ neutral-buffered formalin for $24 \mathrm{~h}$ at room temperature. Paraffin-embedded cross sections ( $2 \mu \mathrm{m}$ to avoid overlap of cells) were cut and mounted on silane-coated glass slides for histological and immunohistochemical examination.

\section{Immunohistochemistry}

Sections were dewaxed in xylene, and rehydrated in graded alcohols and water. Epitope antigen retrieval using microwave and citrate buffer $(\mathrm{pH}=6.0)$ was used for desmin, fibronectin, and ESR1 staining. Endogenous peroxidase activity was blocked with $6 \%(\mathrm{v} / \mathrm{v})$ hydrogen peroxide $\left(\mathrm{H}_{2} \mathrm{O}_{2}\right)$ in water for $10 \mathrm{~min}$. Specific mouse-on-mouse blocking reagent (Vector Labs, Peterborough, UK) was applied for $1 \mathrm{~h}$ where applicable. Sections were incubated overnight at $4{ }^{\circ} \mathrm{C}$ with the primary antibodies against desmin (Mouse monoclonal clone DR33, 1:50 (v/v), Dako, Cambridge, UK), vimentin (Mouse monoclonal, clone LN6, 1:50 (v/v), Chemicon, Millipore, Watford, UK), laminin (Rabbit polyclonal, 1:200 (v/v), Abcam, Cambridge, UK), fibronectin (Rabbit polyclonal, 1:500 (v/v), Abcam), and ESR1 (Mouse monoclonal, clone ER-6F11, Novocastra, Newcastle upon Tyne, UK), and for $1 \mathrm{~h}$ at room temperature for $\alpha$-SMA (clone SMM1, Vector Labs). Biotinylated rabbit anti-mouse or swine anti-rabbit secondary antibody (Dako) was applied at a concentration 1:400 (v/v) for $30 \mathrm{~min}$ at room temperature. Immunoreactivity was demonstrated with 3,3'-diaminobenzidine $/ \mathrm{H}_{2} \mathrm{O}_{2}$ (DAB solution) (Vector Labs). Sections were lightly counterstained with hematoxylin, then dehydrated and cleared in graded alcohol and xylene, and finally covered with glass slips. The immunostaining was assessed for the distribution and intensity using a visual score ( - negative; \pm equivocal, + weak $;+$ moderate, and +++ strong).
Image capture and analysis were performed using Axioplan 2 light microscopy (Carl Zeiss, Gottingen, Germany) and an image capture system. The system was based on a single chip color video camera (Sony DXC-151P, Sony Inc., Japan) connected to a camera adapter (Sony CMA-151P, Sony Inc.) that transmits the image to a Windows-based computer via a Meteor 2 MMC graphics display interface and the Axiovision image analysis software (version 4.0, Carl Zeiss).

\section{Declaration of interest}

The authors declare that there is no conflict of interest that could be perceived as prejudicing the impartiality of the research reported.

\section{Funding}

This work was supported by the University of Leicester and the University Hospitals of Leicester NHS Trust.

\section{Acknowledgements}

We would like to thank Dr lan White (Honorary Reader, MRC Molecular Endocrinology Unit, Department of Cancer Studies and Molecular Medicine, University of Leicester, UK) for his guidance and advice all through the experiment, and for his kind review of the manuscript and his useful comments. We would also like to thank the staff at the Division of Biomedical Services, University of Leicester for their excellent technical assistance.

\section{References}

Azziz R 1989 Adenomyosis: current perspectives. Obstetrics and Gynecology Clinics of North America 16 221-235.

Baskin GB, Smith SM \& Marx PA 2002 Endometrial hyperplasia, polyps, and adenomyosis associated with unopposed estrogen in rhesus monkeys (Macaca mulatta). Veterinary Pathology 39 572-575.

Batra S 1990 Interaction of antiestrogens with binding sites for muscarinic cholinergic drugs and clacium channel blockers in cell membranes. Cancer Chemotherapy and Pharmacology 26 310-312.

Bird CC, McElin TW \& Manalo-Estrella P 1972 The elusive adenomyosis of the uterus-revisited. American Journal of Obstetrics and Gynecology 112 583-593.

Brody JR \& Cunha GR 1989 Histologic, morphometric, and immunocytochemical analysis of myometrial development in rats and mice: I. Normal development. American Journal of Anatomy 186 1-20.

Capetanaki Y, Ngai J \& Lazarides E 1984 Characterization and regulation in the expression of a gene encoding for the intermediate filament protein desmin. PNAS 81 6909-6912.

Cunha GR 1976 Stromal induction and specification of morphogenesis and cytodifferentiation of the epithelia of the mullerian ducts and urogenital sinus during development of the uterus and vagina in mice. Journal of Experimental Zoology 196 361-370.

Cunha GR, Young P \& Brody JR 1989 Role of uterine epithelium in the development of myometrial smooth muscle cells. Biology of Reproduction 40 861-871.

Cunha GR, Battle E, Young P, Brody J, Donjacour A, Hayashi N \& Kinbara H 1992 Role of epithelial-mesenchymal interactions in the differentiation and spatial organization of visceral smooth muscle. Epithelial Cell Biology 1 76-83.

Eide A 1975 The effect of oestradiol on the DNA synthesis in neonatal mouse uterus and cervix. Cell Tissue Research 156 551-555. 
Greaves P \& White IN 2006 Experimental adenomyosis. Best Practice \& Research. Clinical Obstetrics \& Gynaecology 20 503-510.

Green AR, Parrott E, Butterworth M, Jones PS, Greaves P \& White INH 2001 Comparisons of the effects of tamoxifen, toremifene and raloxifene on enzyme induction and gene expression in the ovariectomised rat uterus. Journal of Endocrinology 170 555-564.

Green AR, Edwards RE, Greaves P \& White IN 2003 Comparison of the effect of oestradiol, tamoxifen and raloxifene on nerve growth factoralpha expression in specific neonatal mouse uterine cell types using laser capture microdissection. Journal of Molecular Endocrinology 30 1-11.

Green AR, Styles JA, Parrott EL, Gray D, Edwards RE, Smith AG, Gant TW, Greaves P, Al-Azzawi F \& White IN 2005 Neonatal tamoxifen treatment of mice leads to adenomyosis but not uterine cancer. Experimental and Toxicologic Pathology 56 255-263.

Guttner J 1980 Adenomyosis in mice. Zeitschrift für Versuchstierkunde 22 249-251.

Holderegger C \& Keefer D 1986 The ontogeny of the mouse estrogen receptor: the pelvic region. American Journal of Anatomy 177 285-297.

Horgan K, Cooke E, Hallet MB \& Mansel RE 1986 Inhibition of protein kinase $C$ mediated signal transduction by tamoxifen. Biochemical Pharmacology 35 4463-4465.

Huseby RA \& Thurlow S 1982 Effects of prenatal exposure of mice to 'lowdose' diethylstilbestrol and the development of adenomyosis associated with evidence of hyperprolactinemia. American Journal of Obstetrics and Gynecology 144 939-949.

Iguchi T \& Sato T 2000 Endocrine disruption and developmental abnormalities of female reproduction. American Zoologist 40 402-411.

Jefferson WN, Couse JF, Banks EP, Korach KS \& Newbold RR 2000 Expression of estrogen receptor beta is developmentally regulated in reproductive tissues of male and female mice. Biology of Reproduction 62 310-317.

Korach KS 1994 Insights from the study of animals lacking functional estrogen receptor. Science 266 1524-1527.

Kostrzewska A, Laudanski T \& Batra S 1997 Potent inhibition by tamoxifen of spontaneous and agonist-induced contractions of the human myometrium and intramyometrial arteries. American Journal of Obstetrics and Gynecology 176 381-386.

Kroeger EA \& Brandes LJ 1985 Evidence tat tamoxifen is a histamine antagonist. Biochemical and Biophysical Research Communications 131 750-755.

Kurita T, Cooke PS \& Cunha GR 2001 Epithelial-stromal tissue interaction in paramesonephric (Mullerian) epithelial differentiation. Developmental Biology 240 194-211.

Lam HY 1984 Tamoxifen is a calmodulin antagonist in the activation of cAMP phosphodiesterase. Biochemical and Biophysical Research Communications 118 27-32.

Lazarides E 1980 Intermediate filaments as mechanical integrators of cellular space. Nature 238 249-256.

Li Z, Mericksay M, Agbulut O, Butler-Browne G, Carlsson L, Thornell L-E, Babinet C \& Paulin D 1997 Desmin is essential for the tensile strength and integrity of myofibrls but not for myogenic commitment, differentiation, and fusion of skeletal muscle. Journal of Cell Biology 139 129-144.

Lin Z, Lu M-H, Schultheiss T, Choi J, Holtzer S, DiLullo C, Fischman DA \& Holtzer H 1994 Sequential appearance of muscle-specific proteins in myoblasts as a function of time after cell division: evidence for a conserved myoblast differentiation program in skeletal muscle. Cell Motility and the Cytoskeleton 29 1-19.

Lipschutz A, Iglesias R, Panasevich VI \& Salinas S 1967 Pathological changes induced in the uterus of mice with the prolonged administration of progesterone and 19-nor-contraceptives. British Journal of Cancer 21 160-165.

Lipton A \& Morris ID 1986 Calcium antagonism by the antioestrogen tamoxifen. Cancer Chemotherapy and Pharmacology 18 17-20.

Martin L, Finn CA \& Trinder G 1973 Hypertrophy and hyperplasia in the mouse uterus after oestrogen treatment: an autoradiographic study. Journal of Endocrinology $\mathbf{5 6}$ 133-144.
Matsuda M, Sasabe H, Adachi Y, Suzuki T \& Mori T 2001 Increased invasion activity of endometrial stromal cells and elevated expression of matrix metalloproteinase messenger RNA in the uterine tissues of mice with experimentally induced adenomyosis. American Journal of Obstetrics and Gynecology 185 1374-1380.

McCann TO \& Myers RE 1970 Endometriosis in rhesus monkeys. American Journal of Obstetrics and Gynecology 106 516-523.

Milner DJ, Weitzer G, Tran D, Bradley A \& Capetanaki Y 1996 Disruption of muscle architecture and myocardial degeneration in mice lacking desmin. Journal of Cell Biology 134 1255-1270.

Moggs JG, Ashby J, Tinwell H, Lim FL, Moore DJ, Kimber I \& Orphanides G 2004 The need to decide if all estrogens are intrinsically similar. Environmental Health Perspectives 112 1137-1142.

Mori T \& Nagasawa H 1983 Mechanisms of development of prolactininduced adenomyosis in mice. Acta Anatomica 116 46-54.

Mori T, Nagasawa H \& Takahashi S 1981 The induction of adenomyosis in mice by intrauterine pituitary isografts. Life Sciences 29 1277-1282.

Mori T, Ohta Y \& Nagasawa H 1984 Ultrastructural changes in uterine myometrium of mice with experimentally-induced adenomyosis. Experientia 40 1385-1387.

Mori T, Singtripop T \& Kawashima S 1991 Animal model of uterine adenomyosis: is prolactin a potent inducer of adenomyosis in mice? American Journal of Obstetrics and Gynecology 165 232-234.

Ohta Y, Mori T \& Nagasawa H 1985 Ultrastructural changes of endometrium and myometrium during development of adenomyosis in mice with ectopic pituitary transplants. Zoological Science 2 239-247.

Ostrander PL, Mills KT \& Bern HA 1985 Long-term responses of the mouse uterus to neonatal diethylstilbestrol treatment and to later sex hormone exposure. Journal of the National Cancer Institute 74 121-135.

Park K-Y, Dalakas MC, Semino-Mora C, Lee H-S, Litvak S, Takeda K, Ferrans VJ \& Goldfarb LG 2000 Sporadic cardiac and skeletal myopathy caused by a de nove desmin mutation. Clinical Genetics $\mathbf{5 7}$ 423-429.

Parrott E, Butterworth M, Green A, White IN \& Greaves P 2001 Adenomyosis - a result of disordered stromal differentiation. American Journal of Pathology 159 623-630.

Romberger DJ 1997 Fibronectin. International Journal of Biochemistry \& Cell Biology 29 939-943.

Sapino A, Bosco M, Cassoni P, Castellano I, Arisio R, Cserni G, Dei Tos AP, Fortunati N, Catalano MG \& Bussolati G 2006 Estrogen receptor-beta is expressed in stromal cells of fibroadenoma and phyllodes tumors of the breast. Modern Pathology 19 599-606.

Sasaki T, Fassler R \& Hohenester E 2004 Laminin: the crux of basement membranes assembly. Journal of Cell Biology 164 959-963.

Sato T, Okamura H, Ohta Y, Hayashi S, Takasugi N \& Iguchi T 1992 Estrogen receptor expressin in genital tract of female mice treated neonatally with diethylstilbestrol. In Vivo 6 151-156.

Shah SB, Davis J, Weisleder N, Kostavassili I, McCulloch AD, Ralston E, Capetanaki Y \& Lieber RL 2004 Structural and functional roles of desmin in mouse skeletal muscle during passive deformation. Biophysical Journal 86 2993-3008.

Stumpf WE, Narbaitz R \& Sar M 1980 Estrogen receptors in the fetal mouse. Journal of Steroid Biochemistry 12 55-64.

Suire RA, Goodman DG, Valerioe MG, Fredrickson TN, Strandberg JD, Levitt MH, Lingeman CH, Harshbarger JC \& Dawe CJ 1978 Female reproductive system. In Pathology of Laboratory Animals, vol 2, pp 1051-1262. Eds K Benirschke, FM Garner \& TC Jones. New York: Springer-Verlag.

Yamashita S, Newbold RR, McLachlan JA \& Korach KS 1989 Developmental pattern of estrogen receptor expression in female mouse genital tracts. Endocrinology 125 2888-2896.

Received 15 February 2009

First decision 25 March 2009

Accepted 18 May 2009 